\title{
Universal dynamical decoherence control of noisy single- and multi-qubit systems
}

\author{
Goren Gordon*, Noam Erez ${ }^{\dagger}$, Gershon Kurizki ${ }^{\ddagger}$ \\ Department of Chemical Physics, Weizmann Institute of Science, 76100 Rehovot, Israel
}

\begin{abstract}
In this article we develop, step by step, the framework for universal dynamical control of two-level systems (TLS) or qubits experiencing amplitude- or phase-noise (AN or PN) due to coupling to a thermal bath. A comprehensive arsenal of modulation schemes is introduced and applied to either AN or PN, resulting in completely analogous formulae for the decoherence rates, thus underscoring the unified nature of this universal formalism. We then address the extension of this formalism to multipartite decoherence control, where symmetries are exploited to overcome decoherence.
\end{abstract}

PACS numbers:

\footnotetext{
* goren.gordon@weizmann.ac.il

$\dagger$ nerez@weizmann.ac.il

$\ddagger$ gershon.kurizki@weizmann.ac.il
} 


\section{INTRODUCTION}

In-depth study of the mechanisms of decoherence and disentanglement and their prevention in bipartite or multipartite open systems is an essential prerequisite for applications involving quantum information processing or communications [1]. The present article is aimed at furthering our understanding of these formidable issues, which is scanty at best. It is based on recent progress by our group, as well as others, towards a unified approach to the dynamical control of decoherence and disentanglement. This unified approach culminates in universal formulae allowing design of the required control fields.

The topic of multipartite decoherence has been well-investigated in two limits. One of these is relaxation toward steady-state of one-body coherence of spins, atoms, excitons, quantum dots, etc. that are in contact with a much larger reservoir. The other is the collective decoherence of a small (localized) two-body or many-body system, which typically occurs more rapidly than one-body decoherence $[2,3]$.

By contrast, more general problems of decay of non-local mutual entanglement of two or more small systems are less well understood. This decoherence process may occur on a time scale much shorter than the time for either body to undergo local decoherence, but much larger than the time each takes to become disentangled from its environment. The disentanglement of individual particles from their environment is dynamically controlled by interactions on non-Markovian time-scales, as discussed below [4]. Their disentanglement from each other, however, may be purely Markovian [5-7], in which case the present nonMarkovian approach to dynamical control/prevention is insufficient.

\section{A. Dynamical control of decay and decoherence on non-Markovian time scales}

Quantum-state decay to a continuum or changes in its population via coupling to a thermal bath is known as amplitude noise (AN). It characterizes decoherence processes in many quantum systems, e.g., spontaneous emission of photons by excited atoms [2], vibrational and collisional relaxation of trapped ions [8] and the relaxation of current-biased Josephson junctions [9]. Another source of decoherence in the same systems is proper dephasing or phase noise $(\mathrm{PN})$ [3], which does not affect the populations of quantum states, but randomizes their energies or phases. 
A thoroughly studied approach to suppression of decoherence is the "dynamical decoupling" of the system from the bath [10-18]. In particular, "bang-bang" (BB) pulses have been proposed for stroboscopic suppression of proper dephasing: $\pi$-phase flips of the coupling via strong and sufficiently fast resonant pulses applied to the system [13-15]. The identification of a decoherence-free subspace (DFS), wherein symmetrically degenerate states are decoupled from the bath, constitutes a complementary approach [18-22].

Our group has purported to substantially expand the arsenal of decay and decoherence control. We have presented a universal form of the decay rate of unstable states into any reservoir (continuum), dynamically modified by perturbations with arbitrary time dependence, focusing on non-Markovian time-scales [22-26]. An analogous form has been obtained by us for the dynamically modified rate of proper dephasing [25-27]. Our unified, optimized approach reduces to the BB method in the particular case of proper dephasing or decay via coupling to spectrally symmetric (e.g., Lorentzian or Gaussian) noise baths with limited spectral width (see below). The type of phase modulation advocated for the suppression of coupling to asymmetric baths (e.g., phonon or photon baths with frequency cutoff [28]) is, however, drastically different from the BB method. Other situations to which our approach applies, but not the BB method, include amplitude modulation of the coupling to the continuum, as in the case of decay from quasibound states of a periodically tilted washboard potential [24]: such modulation has been experimentally shown [29] to give rise to either slowdown of the decay (Zeno-like behavior) or its speedup (anti-Zeno-like behavior), depending on the modulation rate.

The theory has been generalized by us to finite temperatures and to qubits driven by an arbitrary time-dependent field, which may cause the failure of the rotating-wave approximation [25]. It has also been extended to the analysis of multi-level systems, where quantum interference between the levels may either inhibit or accelerate the decay [30].

Our general approach [24] to dynamical control of states coupled to an arbitrary "bath" or continuum has reaffirmed the intuitive anticipation that, in order to suppress their decay, we must modulate the system-bath coupling at a rate exceeding the spectral interval over which the coupling is significant. Yet our analysis can serve as a general recipe for optimized design of the modulation aimed at an effective use of the fields for decay and decoherence suppression or enhancement. The latter is useful for the control of chemical reactions [31]. 


\section{B. Control of symmetry-breaking multipartite decoherence}

Symmetry is a powerful means of protecting entangled quantum states against decoherence, since it allows the existence of a decoherence-free subspace or a decoherence-free subsystem [3, 4, 9-11, 13-22, 32-34]. In multipartite systems, this requires that all particles be perturbed by the same environment. In keeping with this requirement, quantum communication protocols based on entangled two-photon states have been studied under collective depolarization conditions, namely, identical random fluctuations of the polarization for both photons [35]. Entangled qubits that reside at the same site or at equivalent sites of the system, e.g. atoms in optical lattices, have likewise been assumed to undergo identical decoherence [4].

Locally-decohering entangled states of two or more particles, such that each particle travels along a different channel or is stored at a different site in the system, may break the state symmetry. A possible consequence of this symmetry breaking is the abrupt "death" of the entanglement [5-7]. Such systems, composed of particles undergoing individual or "local" decoherence, do not possess a natural DFS and thus present more challenging problems insofar as decoherence effects are concerned [36].

Our group has recently addressed these challenges by developing a generalized treatment of multipartite entangled states (MES) decaying into zero-temperature baths and subject to arbitrary external perturbations whose role is to provide dynamical protection from decay and decoherence [30, 37]. Our treatment applies to any difference between the couplings of individual particles to the baths. It does not assume the perturbations to be stroboscopic, i.e. strong or fast enough, but rather to act concurrently with the particle-bath interactions. Our main results are to show that by applying local (selective) perturbations to multilevel particles, i.e. by addressing each level and each particle individually, one can create a decoherence-free system of many entangled qubits. Alternatively, one may reduce the problem of locally decohering MES to that of a single decohering particle, whose dynamical control has been thoroughly investigated [13, 14, 24-26]. On the other hand, the combined effect of dephasing and relaxation (phase and amplitude noises) on MES and its control, which constitute a much more formidable problem, have not yet been studied by us. 


\section{Outline}

In this article we develop, step by step, the framework for universal dynamical control by modulating fields of two-level systems or qubits, aimed at suppressing or preventing their noise, decoherence or relaxation in the presence of a thermal bath. To this end, a comprehensive treatment is developed in Sec. II in a more complete and transparent fashion than its brief sketch in Ref.[25]. Its crux is the derivation of a more general master equation (ME) than in previous treatments of a multilevel, multipartite system, weakly coupled to an arbitrary bath and subject to arbitrary temporal driving or modulation. The present ME, derived by the Nakajima-Zwanzig technique [38, 39], is more general than the ones obtained previously in that it does not invoke the rotating wave approximation and therefore applies at arbitrarily short times or for arbitrarily fast modulations.

Remarkably, when our general ME is applied to either AN or PN in Sec. III, the resulting dynamically-controlled relaxation or decoherence rates obey analogous formulae provided the the corresponding density-matrix (generalized Bloch) equations are written in the appropriate basis. This underscores the universality of our treatment. The choice of an appropriate time-dependent basis allows here to simplify the AN treatment of Ref. [25]. More importantly, it allows us to present a PN treatment that does not describe noise phenomenologically as in Ref. [25], but rather dynamically, starting from the ubiquitous spin-boson Hamiltonian.

We then discuss in Sec. IV, more comprehensively than in previous treatments, the possible modulation arsenal for either AN or PN control. The present formalism is applicable in a natural and straightforward manner to multipartite and/or multilevel systems [37]. It allows us to focus in Sec. V on the ability of symmetries to overcome multipartite decoherence[1821, 34]. Our conclusions are presented in Sec. VI. 


\section{MASTER EQUATION (ME) FOR DYNAMICALLY CONTROLLED SYS- TEMS COUPLED TO THERMAL BATHS}

\section{A. Derivation of the reduced density matrix ME by the Nakajima-Zwanzig} method

We shall consider the most general unitary evolution of a system coupled to a thermal reservoir, governed by the Liouville operator equation (we shall take $\hbar=1$ throughout the rest of the paper):

$$
\begin{gathered}
\dot{\rho}_{\text {tot }}(t)=-i\left[H(t), \rho_{\text {tot }}(t)\right] \equiv-i \mathcal{L}(t) \rho_{\text {tot }}(t), \\
H(t)=H_{0}(t)+H_{I}(t), \\
H_{0}(t) \equiv H_{S}(t)+H_{B}, \\
\mathcal{L}(t)=\mathcal{L}_{S}(t)+\mathcal{L}_{B}+\mathcal{L}_{I}(t)
\end{gathered}
$$

where $\rho_{\text {tot }}$ is the density matrix of the system+reservoir and $H_{S}, H_{B}, H_{I}$ are the Hamiltonians of the system, bath and their interaction, respectively. As usual, $\mathcal{L}$ denotes the Liouville operator, which acts linearly on operators on our Hilbert space. We shall use the notation $H_{0}(t)$ for the "unperturbed" Hamiltonian, assuming weak system-bath coupling, and $\mathcal{L}_{0}(t)$ for its associated Liouville operator.

We seek a master equation for the reduced density matrix of the system alone $\rho \equiv$ $\operatorname{Tr}_{B} \rho_{t o t}$, allowing for arbitrary time dependence of $H(t)$ without resorting to the rotatingwave approximation $[2,3]$. This can be accomplished by the Nakajima-Zwanzig [38-40] projection-operator technique (see also $[41,42]$ ). Let us define $\rho_{B} \equiv Z^{-1} e^{-H_{B} / k_{B} T}$ ( $T$ being the reservoir temperature, $Z$ normalization to unit trace), the projection operator $\mathcal{P}(\cdot) \equiv$ $\operatorname{Tr}_{B}(\cdot) \otimes \rho_{B}$ (satisfying $\mathcal{P}^{2}=\mathcal{P}$ ), and the complementary (projection) operator $\mathcal{Q} \equiv 1-\mathcal{P}$.

In terms of these definitions, Eq.(1) is equivalent to:

$$
\begin{aligned}
& \mathcal{P} \dot{\rho}_{\text {tot }}(t)=-i \mathcal{P} \mathcal{L}(t) \mathcal{P} \rho_{\text {tot }}(t)-i \mathcal{P} \mathcal{L}(t) \mathcal{Q} \rho_{\text {tot }}(t) \\
& \mathcal{Q} \dot{\rho}_{\text {tot }}(t)=-i \mathcal{Q} \mathcal{L}(t) \mathcal{P} \rho_{\text {tot }}(t)-i \mathcal{Q} \mathcal{L}(t) \mathcal{Q} \rho_{\mathrm{tot}}(t)
\end{aligned}
$$


Equation $(4 b)$ is then formally integrated to give:

$$
\mathcal{Q} \rho_{\text {tot }}(t)=-i \int_{0}^{t} \mathcal{K}_{+}(t, \tau) \mathcal{Q} \mathcal{L}(\tau) \mathcal{P} \rho_{\text {tot }}(\tau) d \tau+\mathcal{K}_{+}(t, 0) \mathcal{Q} \rho_{\text {tot }}(0)
$$

where

$$
\mathcal{K}_{+}(t, \tau)=\mathrm{T}_{+} e^{-i \mathcal{Q} \int_{\tau}^{t} \mathcal{L}(s) d s}
$$

$\mathrm{T}_{+}$denoting time-ordering.

If this expression is then plugged into Eq.(4a) we get a non-Markovian ME for $P \rho_{\text {tot }}$ :

$$
\mathcal{P} \dot{\rho}_{\text {tot }}(t)=-i \mathcal{P} \mathcal{L}(t) \mathcal{P} \rho_{\text {tot }}(t)-\int_{0}^{t} \mathcal{P} \mathcal{L}(t) \mathcal{K}_{+}(t, \tau) \mathcal{Q} \mathcal{L}(\tau) \mathcal{P} \rho_{\text {tot }}(\tau) d \tau-i \mathcal{P} \mathcal{L} \mathcal{K}_{+}(t, 0) \mathcal{Q} \rho_{\text {tot }}(0)
$$

which yields a ME for $\rho$ after tracing out the bath.

Rather than apply a perturbative treatment directly to this equation, it is useful to first transform it to a "time-convolutionless" form (TCL) [43-46]. In this form, the memory effect (the presence of $\rho_{\text {tot }}(\tau)$ in the integrand) is transferred to the integration kernel and $\rho(t)$ is taken out of the integral. Only then, is the perturbative expansion (in $\mathcal{L}_{I}$ ) applied.

Formally,

$$
\rho_{\text {tot }}(\tau)=\mathcal{G}_{-}(t, \tau) \rho_{\text {tot }}(t)
$$

where, writing $T_{-}$for anti-chronological ordering:

$$
\mathcal{G}_{-}(t, \tau) \equiv \mathrm{T}_{-} e^{+i \int_{\tau}^{t} \mathcal{L}(s) d s}
$$

Substituting this expression for $\rho_{\text {tot }}(\tau)$ into Eq.(5), one obtains:

$$
\mathcal{Q} \rho_{\text {tot }}(t)=-\int_{0}^{t} \mathcal{K}_{+}(t, \tau) i \mathcal{Q} \mathcal{L}(\tau) \mathcal{P} \mathcal{G}_{-}(t, \tau) d \tau(\mathcal{P}+\mathcal{Q}) \rho_{\text {tot }}(t)+\mathcal{K}_{+}(t, 0) \mathcal{Q} \rho_{\text {tot }}(0)
$$

Collecting all $\mathcal{Q} \rho_{\text {tot }}$ terms on the left, we obtain:

$$
\begin{aligned}
& \mathcal{F}(t) \mathcal{Q} \rho_{\text {tot }}(t)=\{1-\mathcal{F}(t)\} \mathcal{P} \rho_{\text {tot }}(t)+\mathcal{K}_{+}(t, 0) \mathcal{Q} \rho_{\text {tot }}(0) \\
& \mathcal{F}(t)=1+\int_{0}^{t} \mathcal{K}_{+}(t, \tau) i \mathcal{Q} \mathcal{L}(\tau) \mathcal{P} \mathcal{G}_{-}(t, \tau) d \tau \equiv 1+\Sigma(t)
\end{aligned}
$$


Assuming $\mathcal{F}(t)$ can be inverted (which is expected to hold for short times in the weak coupling limit), and writing $\Theta(t)=\mathcal{F}(t)^{-1}$, one obtains the equation:

$$
\mathcal{Q} \rho_{\text {tot }}(t)=\{\Theta(t)-1\} \mathcal{P} \rho_{\text {tot }}(t)+\Theta(t) \mathcal{K}_{+}(t, 0) \mathcal{Q} \rho_{\text {tot }}(0)
$$

Finally, plugging this expression for $\mathcal{Q} \rho_{\text {tot }}$ into Eq.(4a), the formal TCL ME is obtained:

$$
\mathcal{P} \dot{\rho}_{\text {tot }}(t)=-i \mathcal{P} \mathcal{L}(t) \mathcal{P} \rho_{\text {tot }}(t)-i \mathcal{P} \mathcal{L}\{\Theta(t)-1\} \mathcal{P} \rho_{\text {tot }}(t)-i \mathcal{P} \mathcal{L} \Theta(t) \mathcal{K}_{+}(t, 0) \mathcal{Q} \rho_{\text {tot }}(0)
$$

If the initial condition is such that $\mathcal{Q} \rho_{\text {tot }}(0)=0$, so that the last term vanishes (as will indeed be our assumption below), then all memory effects are contained in $\Theta(t)$. In what follows, we shall always assume that

$$
\rho_{\text {tot }}(0)=\rho_{S}(0) \otimes \rho_{B}
$$

so that this condition is fulfilled.

The operators $\mathcal{L}_{S}$ and $\mathcal{L}_{B}$ both commute with $\mathcal{P}$ and $\mathcal{Q}$, and $\mathcal{P} \mathcal{L}_{B}=0$. This implies $\mathcal{P} \mathcal{L} \mathcal{Q}=\mathcal{P} \mathcal{L}_{I} \mathcal{Q}$ (and note that $\left.\Theta-1=\mathcal{Q}(\Theta-1)\right)$. With the notation $\langle\cdot\rangle_{B} \equiv \operatorname{Tr}_{B}\left(\cdot \rho_{B}\right)$, the $\mathrm{ME}$ for the reduced density matrix of the system can be written in the form:

$$
\begin{gathered}
\dot{\rho}(t)=-i\left[\mathcal{L}_{S}+\left\langle\mathcal{L}_{I}\right\rangle_{B}\right] \rho(t)-\Xi(t) \rho(t) \\
\Xi(t)=\left\langle i \mathcal{L}_{I}\{\Theta(t)-1\}\right\rangle_{B}
\end{gathered}
$$

\section{B. Born approximation}

At this point, it is expedient to follow the perturbational method of $[47,48]$. We begin by noting that (cf. Eqs.(10c),(12b)):

$$
\Xi(t)=-i\left\langle\mathcal{L}_{I}\left\{\Sigma(t)+\mathcal{O}\left(\Sigma^{2}\right)\right\}\right\rangle_{B} ; \quad \Sigma(t)=\mathcal{O}\left(\mathcal{L}_{I}\right)
$$

Therefore, to expand Eq.(12b) to second order in $\mathcal{L}_{I}$, we need to evaluate $\mathcal{K}_{+}$and $G$ only to 0 th order! The operator $\mathcal{K}_{+}(t, \tau)$ can be factored:

$$
\mathcal{K}_{+}(t, \tau)=\mathcal{V}_{0}(t, \tau) \mathrm{T}_{+} e^{-i \mathcal{Q} \int_{\tau}^{t} \mathcal{V}_{0}(s, \tau)^{-1} \mathcal{L}_{I}(s) \mathcal{V}_{0}(s, \tau) d s}
$$


where

$$
\mathcal{V}_{0}(t, \tau)=\mathrm{T}_{+} e^{-i \mathcal{Q} \int_{\tau}^{t} \mathcal{L}_{0}(s) d s}
$$

To zeroth order in $\mathcal{L}_{I}, \mathcal{K}_{+}$is just $\mathcal{V}_{0}$. Furthermore, it is important to note that

$$
\mathcal{V}_{0} \mathcal{Q}=\mathcal{Q U}_{0} \mathcal{Q}=\mathcal{Q U}_{0}
$$

where

$$
\mathcal{U}_{0}(t, \tau)=\mathrm{T}_{+} e^{-i \int_{\tau}^{t} \mathcal{L}_{0}(s) d s}
$$

and in Eq.(15a) we have used the fact that $\mathcal{U}_{0}$ commutes with $\mathcal{Q}$. Similarly,

$$
\mathcal{G}_{-}(t, \tau)=\left(1+\mathcal{O}\left(\mathcal{L}_{I}\right)\right) \mathrm{T}_{-} e^{+i \int_{\tau}^{t} \mathcal{L}_{0}(s) d s}=\mathcal{U}_{0}(t, \tau)^{-1}+\mathcal{O}\left(\mathcal{L}_{I}\right)
$$

We have for $\Sigma(t)$ in the Born approximation:

$$
\Sigma(t)=i \int_{0}^{t} \mathcal{V}_{0}(t, \tau) \mathcal{Q} \mathcal{L}_{I}(\tau) \mathcal{P} \mathcal{U}_{0}(t, \tau)^{-1} d \tau
$$

Finally, after making use of Eq.(15a), we get the ME for $\rho$ in the Born approximation,

which implies the neglect of the back-effect of the system on the bath, consistently with the weak-coupling assumption:

$$
\dot{\rho}(t)=-i\left(\mathcal{L}_{S}(t)+\left\langle\mathcal{L}_{I}\right\rangle_{B}\right) \rho(t)-\int_{0}^{t}\left\langle\mathcal{L}_{I}(t) \mathcal{Q} \mathcal{U}_{0}(t, \tau) \mathcal{L}_{I}(\tau) \mathcal{P} \mathcal{U}_{0}(t, \tau)^{-1}\right\rangle_{B} d \tau \rho(t)
$$

for $\mathcal{Q} \rho_{\text {tot }}(0)=0$.

\section{Explicit equations for factorizable interaction Hamiltonians}

We now wish to write the ME explicitly for time-dependent Hamiltonians of the following form [25]:

$$
\begin{aligned}
H(t) & =H_{S}(t)+H_{B}+H_{I}(t), \\
H_{I}(t) & =S(t) B
\end{aligned}
$$


where $H_{S}$ and $H_{B}$ are the system and bath Hamiltonians, respectively; and $H_{I}$, the interaction Hamiltonian, is the product of operators $S, B$ which act on the system (resp. bath) alone. We assume $\left\langle\mathcal{L}_{I}\right\rangle_{B}=0$, by virtue of $\langle B\rangle_{B}=0$. This also implies $\mathcal{P} \mathcal{L}_{I} \mathcal{P}=0$. Equation (18) now simplifies to:

$$
\dot{\rho}(t)=-i \mathcal{L}_{S}(t) \rho(t)-\int_{0}^{t} d \tau\left\langle\mathcal{L}_{I}(t) \mathcal{U}_{0}(t, \tau) \mathcal{L}_{I}(\tau) \mathcal{P} \mathcal{U}_{0}(t, \tau)^{-1}\right\rangle_{B} \rho(t) .
$$

Let us now write out the action of the operator $\mathcal{U}_{0}$ in terms of the unitary evolution operators of the system and bath:

$$
\begin{gathered}
\mathcal{U}_{0}(t, \tau) A=U_{S}(t, \tau) U_{B}(t-\tau) A U_{B}(\tau-t) U_{S}(t, \tau)^{\dagger} \\
U_{S}(t, \tau) \equiv \mathrm{T}_{+} e^{-i \int_{\tau}^{t} H_{S}\left(t^{\prime}\right) d t^{\prime}} \\
U_{B}(t) \equiv e^{-i H_{B} t} .
\end{gathered}
$$

The integrand of the second term in Eq.(20), can now be written explicitly as:

$$
\begin{aligned}
I(t, \tau) & =\operatorname{Tr}_{B}\left[S(t) B, \mathcal{U}_{0}(t, \tau)\left[S(\tau) B, \mathcal{U}_{0}(t, \tau)^{-1} \rho(t) \rho_{B}\right]\right] \\
& =\operatorname{Tr}_{B}\left[S(t) B,\left[\tilde{S}(t, \tau) \tilde{B}(t-\tau), \rho(t) \rho_{B}\right]\right]
\end{aligned}
$$

where $\tilde{S}$ and $\tilde{B}$ are defined as:

$$
\begin{aligned}
\tilde{S}(t, \tau) & \equiv U_{S}(t, \tau) S(\tau) U_{S}(t, \tau)^{\dagger}, \\
\tilde{B}(\tau) & \equiv U_{B}(\tau) B U_{B}(-\tau) .
\end{aligned}
$$

Using the commutativity of $S$ and $B$, and of $\rho_{B}$ and $H_{B}$, as well as the cyclic property of the trace, this gives after some rearrangement:

$$
I(t, \tau)=\langle B \tilde{B}(t-\tau)\rangle_{B}[S(t), \tilde{S}(t, \tau) \rho(t)]+\text { H.c. }
$$

Finally, defining the correlation function for the bath,

$$
\Phi_{T}(t)=\langle B \tilde{B}(t)\rangle_{B}
$$


we obtain the ME for $\rho$ in the Born approximation:

$$
\dot{\rho}(t)=-i\left[H_{S}, \rho(t)\right]+\int_{0}^{t} d \tau\left\{\Phi_{T}(t-\tau)[\tilde{S}(t, \tau) \rho(t), S(t)]+H . c .\right\} .
$$

\section{GENERALIZED BLOCH EQUATIONS}

Having derived the master equation, we focus on two regimes: a two-level system coupled to either an amplitude- or phase-noise (AN or PN) thermal bath. The bath Hamiltonian (in either regime) will be explicitly taken to consist of harmonic oscillators and be linearly coupled to the system (generalizations to other baths and couplings are obvious):

$$
\begin{gathered}
H_{B}=\sum_{\lambda} \omega_{\lambda} a_{\lambda}^{\dagger} a_{\lambda} \\
B=\sum_{\lambda}\left(\kappa_{\lambda} a_{\lambda}+\kappa_{\lambda}^{*} a_{\lambda}^{\dagger}\right) .
\end{gathered}
$$

Here $a_{\lambda}, a_{\lambda}^{\dagger}$ are the annihilation and creation operators of mode $\lambda$, respectively, and $\kappa_{\lambda}$ is the coupling amplitude to mode $\lambda$.

We use different modulation schemes for each regime, namely, dynamical off-resonant fields for the AN regime and time-dependent resonant fields for the $\mathrm{PN}$ regime. We derive the generalized Bloch equations for the two cases.

\section{A. Two-level system coupled to a thermal amplitude-noise bath}

We first consider the AN regime of a two-level system coupled to a thermal bath. We will use off-resonant dynamic modulations, resulting in AC-Stark shifts. The Hamiltonian (Eq. (19a)) then assumes the following form:

$$
\begin{gathered}
H_{S}(t)=\left(\omega_{a}+\delta_{a}(t)\right)|e\rangle\langle e| \\
S(t)=\tilde{\epsilon}(t) \sigma_{x}
\end{gathered}
$$

where $\delta_{a}(t)$ is the dynamical AC-Stark shifts, $\tilde{\epsilon}(t)$ is the time-dependent modulation of the interaction strength, and the Pauli matrix $\sigma_{x}=|e\rangle\langle g|+| g\rangle\langle e|$.

We derive the Bloch equations for the explicit case discussed above. Inserting Eqs. (29)(30) into Eq. (21b) and Eq. (23), we get:

$$
\begin{aligned}
U_{S}(t, \tau) & =e^{-i \omega_{a}(t-\tau)-i \int_{\tau}^{t} d t_{1} \delta_{a}\left(t_{1}\right)}|e\rangle\langle e|+| g\rangle\langle g| \\
\tilde{S}(t, \tau) & =e^{-i \omega_{a}(t-\tau)-i \int_{\tau}^{t} d t_{1} \delta_{a}\left(t_{1}\right)} \tilde{\epsilon}(\tau)|e\rangle\langle g|+\text { H.c. }
\end{aligned}
$$


Plugging this into the ME (26b), we arrive at the following modified Bloch equations:

$$
\begin{aligned}
\dot{\rho}_{e e}=-\dot{\rho}_{g g}= & -R_{e}(t) \rho_{e e}+R_{g}(t) \rho_{g g} \\
\dot{\rho}_{e g}=\dot{\rho}_{g e}^{*}= & -\left\{\left(R(t)+i \Delta_{a}(t)\right)+i\left[\omega_{a}+\delta_{a}(t)\right]\right\} \rho_{e g} \\
& +\left\{R(t)-i \Delta_{a}(t)\right\} \rho_{g e}
\end{aligned}
$$

where

$$
\begin{aligned}
& R(t)=\left[R_{e}(t)+R_{g}(t)\right] / 2 \\
& \Delta_{a}(t)=\Delta_{e}(t)-\Delta_{g}(t) \\
& R_{e(g)}(t) / 2+i \Delta_{e(g)}(t)=\int_{0}^{t} d t^{\prime} \Phi_{T}\left(t-t^{\prime}\right) K_{e(g)}\left(t, t^{\prime}\right) e^{ \pm i \omega_{a}\left(t-t^{\prime}\right)} \\
& K_{e}\left(t, t^{\prime}\right)=K_{g}^{*}\left(t, t^{\prime}\right)=\epsilon(t) \epsilon^{*}\left(t^{\prime}\right) \\
& \epsilon(t)=\tilde{\epsilon}(t) e^{i \int_{0}^{t} d t_{1} \delta_{a}\left(t_{1}\right)}
\end{aligned}
$$

$R_{e(g)}(t)$ is the modified downward (upward) transition rate of the excited (ground) state to the ground (excited) state. Their half-rate contributes to the decoherence rate, and $\Delta_{a}(t)$ is the resonance (transition frequency) shift in energy due to the modified coupling to the bath.

\section{B. Two-level system coupled to thermal phase-noise bath}

Next, we consider the PN regime of a two-level system coupled to thermal bath, where we will use near-resonant fields with time-varying amplitude as our control. The Hamiltonians (Eq. (19a)) then assume the following forms:

$$
\begin{gathered}
H_{S}(t)=\omega_{a}|e\rangle\langle e|+V(t) \sigma_{x} \\
S(t)=\tilde{\epsilon}(t) \sigma_{z}
\end{gathered}
$$

where $V(t)=V_{0}(t) e^{-i \omega_{a} t}+c . c$ is the time-dependent resonant field, with real envelope $V_{0}(t)$, $\tilde{\epsilon}(t)$ is the time-dependent modulation of the interaction strength, $\sigma_{z}=|e\rangle\langle e|-| g\rangle\langle g|$.

Since we are interested in dephasing, phases due to the (unperturbed) energy difference between the levels are immaterial. We eliminate this dependence by moving to the rotating frame. To avoid the need to time-order the propagator of the system Hamiltonian we tilt the rotating frame to the time-dependent basis:

$$
|\uparrow\rangle=\frac{1}{\sqrt{2}}\left(e^{-i \omega_{a} t}|e\rangle+|g\rangle\right) \quad|\downarrow\rangle=\frac{1}{\sqrt{2}}\left(e^{-i \omega_{a} t}|e\rangle-|g\rangle\right)
$$


In this frame, the system and bath Hamiltonians become:

$$
\begin{aligned}
\hat{H}_{S}(t) & =\frac{V_{0}(t)}{2} \hat{\sigma}_{z} \\
\hat{S}(t) & =\tilde{\epsilon}(t) \hat{\sigma}_{x}
\end{aligned}
$$

where^ denotes the rotated and tilted frame, $\hat{\sigma}_{z}=|\uparrow\rangle\langle\uparrow|-| \downarrow\rangle\langle\downarrow|$ and $\hat{\sigma}_{x}=|\uparrow\rangle\langle\downarrow|+| \downarrow\rangle\langle\uparrow|$.

We can now derive the Bloch equations for the PN regime discussed above, and demonstrate their analogy to their AN counterparts (33),(34). To this end we insert Eqs. (43)-(44) into Eq. (21b) and Eq. (23), to get:

$$
\begin{aligned}
U_{S}(t, \tau) & =e^{-i \int_{\tau}^{t} d t_{1} V_{0}\left(t_{1}\right) / 2}|\uparrow\rangle\left\langle\uparrow\left|+e^{i \int_{\tau}^{t} d t_{1} V_{0}\left(t_{1}\right) / 2}\right| \downarrow\right\rangle\langle\downarrow| \\
\tilde{S}(t, \tau) & =e^{-i \int_{\tau}^{t} d t_{1} V_{0}\left(t_{1}\right)} \tilde{\epsilon}(t)|\uparrow\rangle\langle\downarrow|+H . c .
\end{aligned}
$$

Plugging this into the ME (26b), we arrive at the following modified Bloch equations:

$$
\begin{aligned}
& \dot{\rho}_{\uparrow \uparrow}=-\dot{\rho}_{\downarrow \downarrow}=-R_{\uparrow}(t) \rho_{\uparrow \uparrow}+R_{\downarrow}(t) \rho_{\downarrow \downarrow} \\
& \dot{\rho}_{\uparrow \downarrow}=\dot{\rho}_{\downarrow \uparrow}^{*}=-\left\{\left(R(t)+i \Delta_{a}(t)\right)+i V_{0}(t) / 2\right\} \rho_{\uparrow \downarrow}+\left\{R(t)-i \Delta_{a}(t)\right\} \rho_{\downarrow \uparrow},
\end{aligned}
$$

where

$$
\begin{aligned}
& R(t)=\left[R_{\uparrow}(t)+R_{\downarrow}(t)\right] / 2 \\
& \Delta_{a}(t)=\Delta_{\uparrow}(t)-\Delta_{\downarrow}(t) \\
& R_{\uparrow(\downarrow)}(t) / 2+i \Delta_{\uparrow(\downarrow)}(t)=\int_{0}^{t} d t^{\prime} \Phi_{T}\left(t-t^{\prime}\right) K_{\uparrow(\downarrow)}\left(t, t^{\prime}\right) \\
& K_{\uparrow}\left(t, t^{\prime}\right)=K_{\downarrow}^{*}\left(t, t^{\prime}\right)=\epsilon(t) \epsilon^{*}\left(t^{\prime}\right) \\
& \epsilon(t)=\tilde{\epsilon}(t) e^{i \int_{0}^{t} d t_{1} V_{0}\left(t_{1}\right)} .
\end{aligned}
$$

As can be clearly seen, these modified Bloch equations are completely analogous to their AN counterparts, Eqs. (33),(34), provided we change the basis as follows:

$$
e \leftrightarrow \uparrow \quad g \leftrightarrow \downarrow
$$

Despite their analogy, Eqs. (37) and (51) are not identical, due to the use of the rotating frame in the PN case. Nevertheless, this analogy underscores the universality of our approach. 


\section{Spectral domain representation}

For both AN and PN regime, one can have a more insightful representation of the modified rates by transforming them to the frequency domain. For the long time limits (see Sec. V), one arrives at the form

$$
\begin{aligned}
& R_{e(g)}(t)=2 \pi \int_{-\infty}^{\infty} d \omega G_{T}\left( \pm\left(\omega_{a}+\omega\right)\right) F_{t}(\omega) \\
& R_{\uparrow(\downarrow)}(t)=2 \pi \int_{-\infty}^{\infty} d \omega G_{T}( \pm \omega) F_{t}(\omega)
\end{aligned}
$$

where the difference is due to the fact that we used the rotating and tilted frame in the PN regime. Here $G_{T}(\omega)$ is the temperature-dependent bath coupling spectrum given by

$$
G_{T}(\omega)=(2 \pi)^{-1} \int_{-\infty}^{\infty} d t \Phi_{T}(t) e^{i \omega t} .
$$

Introducing the control-field fluence $Q(t)$, the spectral modulation $F_{t}(\omega)$ can be normalized to unity:

$$
\begin{gathered}
Q(t)=\int_{0}^{t} d \tau|\epsilon(\tau)|^{2}, \\
F_{t}(\omega)=\frac{\left|\epsilon_{t}(\omega)\right|^{2}}{Q_{(}(t)},
\end{gathered}
$$

where

$$
\epsilon_{t}(\omega)=\frac{1}{\sqrt{2 \pi}} \int_{0}^{t} d \tau \epsilon(\tau) e^{i \omega \tau}
$$

is the finite-time Fourier transform of $\epsilon(t)$.

One can consider a more specific scenario, namely, coupling to zero-temperature $(T=0)$ AN bath. The effects of the bath then amount to the decay of the excited state's population, which can be written as:

$$
P_{e}(t)=\exp \left[-R_{e}(t) Q(t)\right]
$$

\section{MODULATION ARSENAL FOR AN AND PN}

Any modulation with quasi-discrete, finite spectrum is deemed quasiperiodic, implying that it can be expanded as

$$
\epsilon(t)=\sum_{k} \epsilon_{k} e^{-i \nu_{k} t}
$$


where $\nu_{k}(k=0, \pm 1, \ldots)$ are arbitrary discrete frequencies such that

$$
\left|\nu_{k}-\nu_{k^{\prime}}\right| \geq \Omega \quad \forall k \neq k^{\prime},
$$

where $\Omega$ is the minimal spectral interval.

One can define the long-time limit of the quasi-periodic modulation, when

$$
\Omega t \gg 1 \text { and } t \gg t_{c}
$$

where $t_{c}$ is the bath-memory (correlation) time, defined as the inverse of the largest spectral interval over which $G_{T}(\omega)$ and $G_{T}(-\omega)$ change appreciably near the relevant frequencies $\omega_{a}+\nu_{k}$. In this limit, the fluence is given by

$$
Q(t) \approx \epsilon_{c} t \quad \epsilon_{c}=\sum_{k}\left|\epsilon_{k}\right|^{2},
$$

resulting in the average decay rate:

$$
\begin{aligned}
& R_{e}=2 \pi \sum_{k}\left|\lambda_{k}\right|^{2} G\left(\omega_{a}+\nu_{k}\right), \\
& \lambda_{k}=\epsilon_{k} / \epsilon_{c} .
\end{aligned}
$$

\section{A. Phase modulation (PM) of the coupling}

\section{Monochromatic perturbation}

Let

$$
\epsilon(t)=\epsilon_{0} e^{-i \Delta t} .
$$

Then

$$
R_{e}=2 \pi G_{T}\left(\omega_{a}+\Delta\right),
$$

where $\Delta=$ const. is a frequency shift, induced by the AC Stark effect (in the case of atoms) or by the Zeeman effect (in the case of spins). In principle, such a shift may drastically enhance or suppress $R$ relative to the Golden - Rule decay rate, i.e. the decay rate without any perturbation

$$
R_{\mathrm{GR}}=2 \pi G_{T}\left(\omega_{a}\right) .
$$

Equation (69) provides the maximal change of $R$ achievable by an external perturbation, since it does not involve any averaging (smoothing) of $G(\omega)$ incurred by the width of $F_{t}(\omega)$ : 
the modified $R$ can even vanish, if the shifted frequency $\omega_{a}+\Delta$ is beyond the cutoff frequency of the coupling, where $G(\omega)=0$ (Figure 1a). This would accomplish the goal of dynamical decoupling [10-18]. Conversely, the increase of $R$ due to a shift can be much greater than that achievable by repeated measurements, i.e. the anti-Zeno effect $[22,23,27,49]$. In practice, however, AC Stark shifts are usually small for (cw) monochromatic perturbations, whence pulsed perturbations should often be used, resulting in multiple $\nu_{k}$ shifts, as per Eq. (66).

\section{Impulsive phase modulation}

Let the phase of the modulation function periodically jump by an amount $\phi$ at times $\tau, 2 \tau, \ldots$. Such modulation can be achieved by a train of identical, equidistant, narrow pulses of nonresonant radiation, which produce pulsed AC Stark shifts of $\omega_{a}$. Now

$$
\epsilon(t)=e^{i[t / \tau] \phi},
$$

where $[\ldots]$ is the integer part. One then obtains that

$$
\begin{gathered}
Q(t)=t, \quad \epsilon_{c}=1, \\
F_{n \tau}(\omega)=\frac{2 \sin ^{2}(\omega \tau / 2) \sin ^{2}[n(\phi+\omega \tau) / 2]}{\pi n \tau \omega^{2} \sin ^{2}[(\phi+\omega \tau) / 2]} .
\end{gathered}
$$

The excited-state decay, according to equation (61), has then the form (at $t=n \tau$ )

$$
P_{e}(n \tau)=\exp \left[-R_{e}(n \tau) n \tau\right]
$$

where $R_{e}(n \tau)$ is defined by Eqs. (55) and (73).

For sufficiently long times (Eq. (64)) one can use Eq. (66), with

$$
\nu_{k}=\frac{2 k \pi}{\tau}-\frac{\phi}{\tau}, \quad\left|\lambda_{k}\right|^{2}=\frac{4 \sin ^{2}(\phi / 2)}{(2 k \pi-\phi)^{2}}
$$

For small phase shifts, $\phi \ll 1$, the $k=0$ peak dominates,

$$
\left|\lambda_{0}\right|^{2} \approx 1-\frac{\phi^{2}}{12}
$$

whereas

$$
\left|\lambda_{k}\right|^{2} \approx \frac{\phi^{2}}{4 \pi^{2} k^{2}} \quad(k \neq 0)
$$


In this case one can retain only the $k=0$ term in Eq. (66), unless $G(\omega)$ is changing very fast with frequency. Then the modulation acts as a constant shift, (Fig. 1a)

$$
\Delta=-\phi / \tau
$$

As $|\phi|$ increases, the difference between the $k=0$ and $k=1$ peak heights diminishes, vanishing for $\phi= \pm \pi$. Then

$$
\left|\lambda_{0}\right|^{2}=\left|\lambda_{1}\right|^{2}=4 / \pi^{2}
$$

i.e., $F_{t}(\omega)$ for $\phi= \pm \pi$ contains two identical peaks symmetrically shifted in opposite directions (Figure 1b) [the other peaks $\left|\lambda_{k}\right|^{2}$ decrease with $k$ as $(2 k-1)^{-2}$, totaling 0.19].

The foregoing features allow one to adjust the modulation parameters for a given scenario to obtain an optimal decrease or increase of $R$. Thus, the phase-modulation (PM) scheme with a small $\phi$ is preferable near a continuum edge (Figure 1a,b), since it yields a spectral shift in the required direction (positive or negative). The adverse effect of $k \neq 0$ peaks in $F_{t}(\omega)$ then scales as $\phi^{2}$ and hence can be significantly reduced by decreasing $|\phi|$. On the other hand, if $\omega_{a}$ is near a symmetric peak of $G(\omega), R$ is reduced more effectively for $\phi \simeq \pi$, as in Refs. [11, 32], since the main peaks of $F_{t}(\omega)$ at $\omega_{0}$ and $\omega_{1}$ then shift stronger with $\tau^{-1}$ than the peak at $\omega_{0}=-\phi / \tau$ for $\phi \ll 1$.

(a)

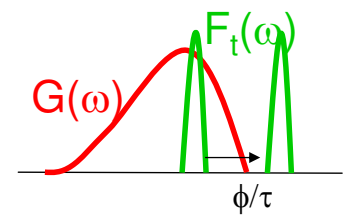

(b)

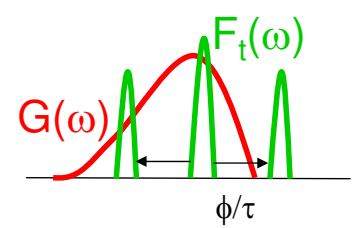

(c)

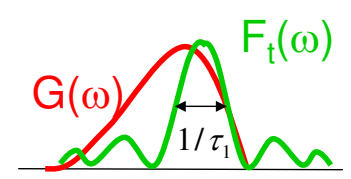

FIG. 1: Spectral representation of the bath coupling, $G(\omega)$, and the modulation, $F_{t}(\omega)$. (a) Monochromatic modulation, or impulsive phase modulation, with small phase shifts, $\phi \ll 1$, and $1 / \tau$ repetition rate. (b) Impulsive phase modulation, ( $\pi$-pulses), $\phi=\pi$. (c) On-off modulation, with $1 / \tau_{1}$ repetition rate for $\tau_{1} \ll \tau_{0}$. 


\section{B. Amplitude modulation (AM) of the coupling}

Amplitude modulation (AM) of the coupling may be applicable to certain AN or PN scenarios. It arises, e.g., for radiative-decay modulation due to atomic motion through a high- $Q$ cavity or a photonic crystal $[50,51]$ or for atomic tunneling in optical lattices with time-varying lattice acceleration $[29,52]$.

\section{On-off modulation}

The simplest form of AM is to let the coupling be turned on and off periodically, for the time $\tau_{1}$ and $\tau_{0}-\tau_{1}$, respectively, i.e.,

$$
\epsilon(t)=\left\{\begin{array}{l}
1 \text { for } n \tau_{0}<t<n \tau_{0}+\tau_{1} \\
0 \text { for } n \tau_{0}+\tau_{1}<t<(n+1) \tau_{0}
\end{array}\right.
$$

$(n=0,1, \ldots)$. Now $Q(t)$ in $(61)$ is the total time during which the coupling is switched on, whereas

$$
F_{n \tau_{0}}(\omega)=\frac{2 \sin ^{2}\left(\omega \tau_{1} / 2\right) \sin ^{2}\left(n \omega \tau_{0} / 2\right)}{\pi n \tau_{1} \omega^{2} \sin ^{2}\left(\omega \tau_{0} / 2\right)}
$$

so that

$$
P\left(n \tau_{0}\right)=\exp \left[-R\left(n \tau_{0}\right) n \tau_{1}\right]
$$

where $R\left(n \tau_{0}\right)$ is given by Eqs. (55) and (81). This case is also covered by (66), where the parameters are now found to be

$$
\epsilon_{c}^{2}=\frac{\tau_{1}}{\tau_{0}}, \quad \nu_{k}=\frac{2 k \pi}{\tau_{0}}, \quad\left|\lambda_{k}\right|^{2}=\frac{\tau_{1}}{\tau_{0}} \operatorname{sinc}^{2}\left(\frac{k \pi \tau_{1}}{\tau_{0}}\right) .
$$

It is instructive to consider the limit wherein $\tau_{1} \ll \tau_{0}$ and $\tau_{0}$ is much greater than the correlation time of the continuum, i.e., $G(\omega)$ does not change significantly over the spectral intervals $\left(2 \pi k / \tau_{0}, 2 \pi(k+1) / \tau_{0}\right)$. In this case one can approximate the sum (66) by the integral (55) with

$$
F_{t}(\omega) \approx\left(\tau_{1} / 2 \pi\right) \operatorname{sinc}^{2}\left(\omega \tau_{1} / 2\right)
$$

characterized by the spectral broadening $\sim 1 / \tau_{1}$ (figure 1c). Then equation (55) for $R$ reduces to that obtained when ideal projective measurements are performed at intervals $\tau_{1}$ [23]. Thus the AM on-off coupling scheme can imitate measurement-induced (dephasing) effects on quantum dynamics, if the interruption intervals $\tau_{0}$ exceed the correlation time of the continuum. 


\section{MULTIPARTITE DECOHERENCE CONTROL}

Multipartite decoherence control, for many qubits coupled to thermal baths, is a much more challenging task than single-qubit control since: (i) entanglement between the qubits is typically more vulnerable and more rapidly destroyed by the environment than single qubit coherence [5-7]; (ii) the possibility of cross-decoherence, whereby qubits are coupled to each other through the baths, considerably complicates the control. We have recently analyzed this situation and extended $[30,37]$ the decoherence control approach of Sec. II-IV to multipartite scenarios, where the qubits are either coupled to zero-temperature baths or undergoing proper dephasing.

\section{A. Multipartite AN control by off-resonant modulation: singly excited systems} coupled to $T=0$ baths

The decay of a singly excited multi-qubit system (under amplitude noise) to the ground state, in the presence of off-resonant modulating fields is described by the following relaxation matrix $[30,37]$ :

$$
\begin{aligned}
& J_{j j^{\prime}}(t)=2 \pi \int_{-\infty}^{\infty} d \omega G_{j j^{\prime}}(\omega) F_{t, j j^{\prime}}(\omega) \\
& G_{j j^{\prime}}(\omega)=\hbar^{-2} \sum_{k} \mu_{k, j} \mu_{k, j^{\prime}}^{*} \delta\left(\omega-\omega_{k}\right) \\
& F_{t, j j^{\prime}}(\omega)=\epsilon_{t, j}^{*}\left(\omega-\omega_{j}\right) \epsilon_{t, j^{\prime}}\left(\omega-\omega_{j^{\prime}}\right)
\end{aligned}
$$

Here $G_{j j^{\prime}}(\omega)$ is the coupling spectrum matrix given by nature and $F_{t, j j^{\prime}}(\omega)$ is the dynamical modulation matrix, which we design at will to suppress the decoherence. The diagonal elements of the decoherence matrix are the time-integrated individual qubits' decay rates, while the off-diagonal elements are the cross-relaxation rates, pertaining to the coupling of the different qubits through the bath: virtual emission into the bath by qubit $j$ and its virtual reabsorption by qubit $j^{\prime}$.

As an example, we may control the relaxation matrix elements by local (qubit-addressing) impulsive phase modulation, (see Sec. IV A 2), described by

$$
\epsilon_{t, j}(\omega)=\frac{\left(e^{i \omega \tau_{j}}-1\right)\left(e^{i\left(\phi_{j}+\omega \tau_{j}\right)\left[t / \tau_{j}\right]}-1\right)}{i \omega\left(e^{i\left(\phi_{j}+\omega \tau_{j}\right)}-1\right)} .
$$


Here [...] denote the integer part, $\tau_{j}$ and $\phi_{j}$ are the pulse duration and the phase change for particle $j$, respectively. In the limit of weak pulses, of area $\left|\phi_{j}\right| \ll \pi$, Eq. (88) yields $\epsilon_{t, j}(\omega) \cong \epsilon_{t, j} \delta\left(\omega-\Delta_{j}\right)$, where $\Delta_{j}=\phi_{j} / \tau_{j}$ is the effective spectral shift caused by the pulses.

One can define the fidelity, $F(t)$, total excitation probability, $F_{p}(t)$, and the autocorrelation function, $F_{c}(t)$ as follows:

$$
\begin{aligned}
& F(t)=\operatorname{Tr}_{\{j\}}(\rho(0) \rho(t)) \\
& F_{p}(t)=\operatorname{Tr}_{\left\{j^{\prime} \neq j\right\}}\left({ }_{j}\langle e|\rho(t)| e\rangle_{j}\right) \\
& F_{c}(t)=F(t) / F_{p}(t)
\end{aligned}
$$

where $\operatorname{Tr}_{\{j\}}$ denotes tracing over all qubits. In the absence of dynamical control, the autocorrelation decays much faster than the total excitation probability, and is much more sensitive to the asymmetry between local particle-bath couplings.

Thus, for initial Bell singlet and triplet states, which do not experience cross-decoherence but only different local decoherence rates, we find:

$$
\begin{gathered}
|\Psi(0)\rangle=1 / \sqrt{2}\left(|g\rangle_{A}|e\rangle_{B} \pm|e\rangle_{A}|g\rangle_{B}\right) \\
F_{p}(t)=\left(e^{-2 J_{A}(t)}+e^{-2 J_{B}(t)}\right) / 2 \\
F_{c}(t)=(1+C(t)) / 2=1 / 2+e^{-\Delta J(t)} /\left(1+e^{-2 \Delta J(t)}\right) \\
\Delta J(t)=J_{A}(t)-J_{B}(t)
\end{gathered}
$$

where $C(t)$ is the concurrence [53].

Without any modulations, decoherence in this scenario has no inherent symmetry. Our point is that one can symmetrize the decoherence by appropriate modulations. The key is that different, "local", phase-locked modulations applied to the individual particles, according to Eq. (87), can be chosen to cause controlled interference and/or spectral shifts between the particles' couplings to the bath. The $F_{t, j j^{\prime}}(\omega)$ matrices (cf.(87)) can then satisfy $2 N$ requirements at all times and be tailored to impose the advantageous symmetries described below. By contrast, a "global" (identical) modulation, characterized by $F_{t, j j^{\prime}}(\omega)=\left|\epsilon_{t}(\omega)\right|^{2}$, is not guaranteed to satisfy $N \gg 1$ symmetrizing requirements at all times (Fig. 2a).

The most desirable symmetry is that of identically coupled particles (ICP), which would emerge if all the modulated particles could acquire the same dynamically modified decoherence and cross-decoherence yielding the following $N \times N$ fully symmetrized decoherence 
(a)
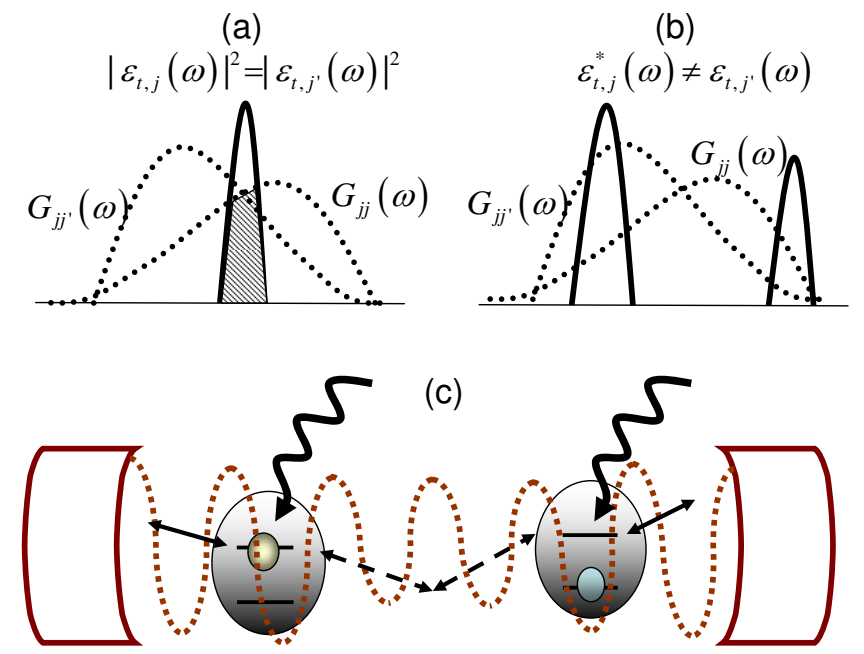

FIG. 2: Two two-level particles in a cavity, coupled to the cavity modes (thin lines) and subject to local control fields (thick lines). (a,b) Frequency domain overlap of coupling spectrum (dotted) and modulation matrix elements(solid), resulting in modified decoherence matrix elements (shaded), for: (a) global modulation (ICP symmetry), (b) cross-decoherence elimination (IIP symmetry). (c) General modulation scheme.

matrix

$$
J_{j j^{\prime}}^{\mathrm{ICP}}(t)=r(t) \quad \forall j, j^{\prime}
$$

ICP would then give rise to a $(N-1)$-dimensional decoherence-free subspace: the entire single-excitation sector less the totally symmetric entangled state. An initial state in this DFS [19] would neither lose its population nor its initial correlations (or entanglement).

Unfortunately, it is generally impossible to ensure this symmetry, since it amounts to satisfying $N(N-1) / 2$ conditions using $N$ modulating fields. Even if we accidentally succeed with $N$ particles, the success is not scalable to $N+1$ or more particles. Moreover, the ability to impose the ICP symmetry by local modulation fails completely if not all particles are coupled to all other particles through the bath, i.e. if some $G_{j j^{\prime}}(\omega)$ elements vanish.

A more limited symmetry that we may ensure for $N$ qubits is that of independent identical particles (IIP). This symmetry is formed when spectral shifts and/or interferences imposed by $N$ modulations cause the $N$ different qubits to acquire the same single-qubit decoherence $r(t)$ and experience no cross-decoherence. To this end, we may choose $\epsilon_{t, j}(\omega) \simeq \epsilon_{t, j} \delta\left(\omega-\Delta_{j}\right)$. We shall deal with $N$ identical qubits, and set $\omega_{j} \equiv \omega_{0}$. We also require that at any chosen time $t=T$, the AC Stark shifts satisfy $\int_{0}^{T} d \tau \delta_{j}(\tau)=2 \pi m$, where $m=0, \pm 1, \ldots$. This 
requirement ensures that modulations only affect the decoherence matrix (85), but do not change the relative phases of the entangled qubits when their MES is probed or manipulated by logic operations at $t=T$.

The spectral shifts $\Delta_{j}$ can be different enough to couple each particle to a different spectral range of bath modes so that their cross-coupling vanishes:

$$
J_{j j^{\prime}}(t)=2 \pi \epsilon_{t, j}^{*} \epsilon_{t, j^{\prime}} \int d \omega G_{j j^{\prime}}\left(\omega_{0}+\omega\right) \delta\left(\omega-\Delta_{j}\right) \delta\left(\omega-\Delta_{j^{\prime}}\right) \rightarrow 0 .
$$

Here, the vanishing of $G_{j j^{\prime}}(\omega)$ for some $j, j^{\prime}$ is not a limitation. The $N$ single-particle decoherence rates can be equated by an appropriate choice of $N$ parameters $\left\{\Delta_{j}\right\}$ :

$$
J_{j j^{\prime}}^{\mathrm{IIP}}(t)=2 \pi\left|\epsilon_{t, j}\right|^{2} G_{j j}\left(\omega_{0}+\Delta_{j}\right)=\delta_{j j^{\prime}} r(t)
$$

where $\delta_{j j^{\prime}}$ is Kronecker's delta (Fig. 1b). The IIP symmetry results in complete correlation preservation, i.e. $F_{c}(t)=1$, but still permits excited-state population loss, $F_{p}(t)=e^{-2 \operatorname{Re}\{r(t)\}}$ (Fig. 3). If the single-particle $r(t)$ may be dynamically suppressed, i.e. if the spectrally shifted bath response $G_{j j}\left(\omega_{j}+\Delta_{j}\right)$ is small enough, this $F_{p}(t)$ will be kept close to 1 .

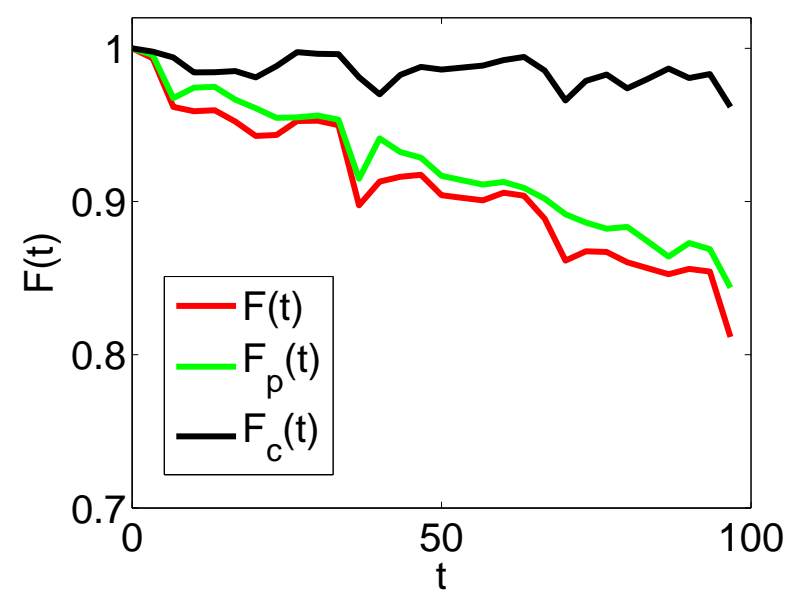

FIG. 3: Fidelity of the IIP symmetry for two TLS coupled to zero-temperature baths. The initial state is entalged, $|\psi(0)\rangle=1 / \sqrt{2}\left(|g\rangle_{A}|e\rangle_{B}+|e\rangle_{A}|g\rangle_{B}\right)$.

\section{B. Multipartite PN control by resonant modulation}

One can describe phase-noise, or proper dephasing, by a stochastic fluctuation of the excited-state energy, $\omega_{a} \rightarrow \omega_{a}+\delta_{r}(t)$, where $\delta_{r}(t)$ is a stochastic variable with zero mean, 
$\langle\delta(t)\rangle=0$, and $\left\langle\delta(t) \delta\left(t^{\prime}\right)\right\rangle=\Phi^{P}\left(t-t^{\prime}\right)$ is the second moment. For multipartite systems, where each qubit can undergo different proper dephasing, $\delta_{j}(t)$, one has an additional second moment for the cross-dephasing, $\left\langle\delta_{j}(t) \delta_{j^{\prime}}\left(t^{\prime}\right)\right\rangle=\Phi_{j j^{\prime}}^{P}\left(t-t^{\prime}\right)$. A general treatment of multipartite systems undergoing this type of proper dephasing is given in Ref. [30]. Here we give the main results for the case of two qubits.

Let us take two TLS, or qubits, which are initially prepared in a Bell state. We wish to obtain the conditions that will preserve it. In order to do that, we change to the Bell basis, which is given by

$$
\begin{aligned}
& \left|B_{1,2}\right\rangle=1 / \sqrt{2} e^{i \omega_{a} t}\left(|e\rangle_{1}|g\rangle_{2} \pm|g\rangle_{1}|e\rangle_{2}\right) \\
& \left|B_{3,4}\right\rangle=1 / \sqrt{2}\left(e^{i 2 \omega_{a} t}|e\rangle_{1}|e\rangle_{2} \pm|g\rangle_{1}|g\rangle_{2}\right) .
\end{aligned}
$$

For an initial Bell-state $\overline{\boldsymbol{\rho}}_{l}(0)=\left|B_{l}\right\rangle\left\langle B_{l}\right|$, where $l=1 \ldots 4$, one can then obtain the fidelity, $F_{l}(t)=\left\langle B_{l}\left|\overline{\boldsymbol{\rho}}_{l}(t)\right| B_{l}\right\rangle$, as:

$$
F_{l}(t)=\cos \left(\phi_{ \pm}(t)\right) \operatorname{Re}\left[e^{i \phi_{ \pm}(t)}\left(1-\frac{1}{2} \sum_{j j^{\prime}} J_{j j^{\prime}, l}^{P}(t)\right)\right]
$$

where

$$
\begin{gathered}
\phi_{j}(t)=2 \int_{0}^{t} d \tau V_{0, j}(\tau) \\
J_{j j^{\prime}, l}^{P}(t)=2 \pi \int_{-\infty}^{\infty} d \omega G_{j j^{\prime}}^{P}(\omega) F_{t, j j^{\prime}, l}(\omega) \\
G_{j j^{\prime}}^{P}(\omega)=\int_{-\infty}^{\infty} d t \Phi_{j j^{\prime}}^{P}(t) e^{i \omega t} \\
F_{t, j j, l}(\omega)=\left|\epsilon_{t, j}(\omega)\right|^{2} \\
F_{t, j j^{\prime}, 3}(\omega)=-F_{t, j j^{\prime}, 1}(\omega)=\epsilon_{t, j}^{*}(\omega) \epsilon_{t, j^{\prime}}^{*}(\omega) \\
F_{t, j j^{\prime}, 4}(\omega)=-F_{t, j j^{\prime}, 2}(\omega)=\epsilon_{t, j}(\omega) \epsilon_{t, j^{\prime}}^{*}(\omega)
\end{gathered}
$$

where $V_{0, j}(t)$ is the amplitude of the resonant field applied on qubit $j, \phi_{ \pm}(t)=\left(\phi_{1}(t) \pm\right.$ $\left.\phi_{2}(t)\right) / 2$ and the $\phi_{+}$corresponds to $k=1,3$ and $\phi_{-}$to $k=2,4$. Expressions (101)-(107) provide our recipe for minimizing the Bell-state fidelity losses. They hold for any dephasing time-correlations and arbitrary modulation.

One can choose between two modulation schemes, depending on our goals. When one wishes to preserve and initial quantum state, one can equate the modified dephasing and cross-dephasing rates of all qubits, $J_{j j^{\prime}, l}(t)=J(t)$. This results in complete preservation of the singlet only, i.e. $F_{2}(t)=1$, for all $t$, but reduces the fidelity of the triplet state. On 
the other hand, if one wishes to equate the fidelity for all initial states, one can eliminate the cross-dephasing terms, by applying different modulations to each qubit (Fig. 4), causing $F_{t, j j^{\prime}, l}(\omega)=0 \forall j \neq j^{\prime}$. This requirement can be important for quantum communication schemes.

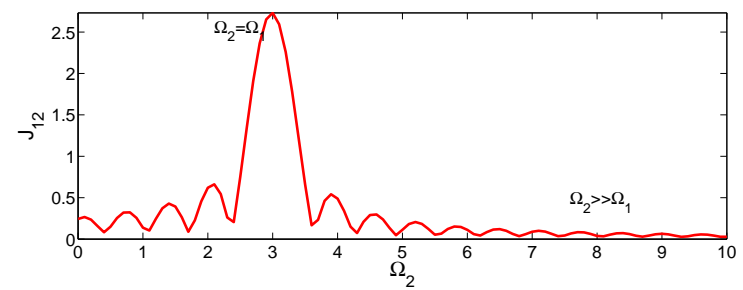

FIG. 4: Cross-decoherence as a function of local modulation. Here two qubits are modulated by continuous resonant fields, with amplitudes $\Omega_{1,2}$. The cross-decoherence decays as the two qubits' modulations become increasingly different. The bath parameters are $\Phi_{T}(t)=e^{-t / t_{c}}$, where $t_{c}=0.5$ is the correlation time; and $\Omega_{1}=3$.

\section{CONCLUSIONS}

In this paper we have expounded our universal approach to the dynamical control of qubits subject to $\mathrm{AN}$ and $\mathrm{PN}$, by either off- or on-resonant modulating fields, respectively. It is based on a general non-Markovian master equation valid for weak system-bath coupling and arbitrary modulations, since it does not invoke the rotating wave approximation. The resulting universal convolution formulae provide intuitive clues as to the optimal tailoring of modulation and noise spectra. Our analysis of multiple, field-driven, qubits which are coupled to partly correlated or independent baths or undergo locally varying random dephasing has resulted in the universal formula (85) for coupling to zero-temperature bath, and (101) for Bell-state preservation under local proper dephasing.

Our general analysis allows one to come up with an optimal choice between global and local control, based on the observation that the maximal suppression of decoherence is not necessarily the best one. Instead, we demand an optimal phase-relation between different, but synchronous local modulations of each particle. The merits of local vs. global modulations have been shown to be essentially twofold:

- Local modulation can effectively decorrelate the different proper dephasings of the 
multiple TLS, resulting in equal dephasing rates for all states. For two TLS, we have shown that the singlet and triplet Bell-states acquire the same dynamicallymodified dephasing rate. This should be beneficial compared to the standard global "Bang-Bang" ( $\pi$-phase flips) if both states are used (intermittently) for information transmission or storage.

- For different couplings to a zero-temperature bath, one can better preserve any initial state by using local modulation which can reduce the decay as well as the mixing with other states, than by using global modulation. It was shown that local modulation which eliminates the cross-decoherence terms, increases the fidelity more than the global modulation alternative. For two TLS, it was shown that local modulation better preserves an initial Bell-state, whether a singlet or a triplet, compared to global $\pi$-phase "parity kicks".

Acknowledgement. We acknowledge the support of the EC (SCALA NoE).

[1] M.A. Nielsen and I.L. Chuang. Quantum Computation and Quantum Information. Cambridge University Press, Cambridge, UK, 2000.

[2] C. Cohen-Tannoudji, J. Dupont-Roc, and G. Grynberg. Atom-Photon Interactions. Wiley, New York, 1992.

[3] M. O. Scully and M. S. Zubairy. Quantum Optics. Cambridge University Press, Cambridge, 1997.

[4] V.M. Akulin, A. Sarfati, G. Kurizki, and S. Pellegrin, editors. Decoherence, Entanglement and Information Protection in Complex Quantum Systems, volume 189 of NATO Science Series II: Mathematics, Physics and Chemistry. Springer, 2005.

[5] S. Bandyopadhyay and D. A. Lidar. Entangling capacities of noisy two-qubit hamiltonians. Phys. Rev. A, 70:010301, 2004.

[6] T. Yu and J. H. Eberly. Finite-time disentanglement via spontaneous emission. Phys. Rev. Lett., 93(14):140404, 2004.

[7] T. Yu and J. H. Eberly. Opt. Comm. XX, in press (2006) and arXiv: quant-ph/0602196. 
[8] C. A. Sackett, D. Kielpinski, B. E. King, C. Langer, V. Meyer, C.J. Myatt, M. Rowe, Q. A. Turchette, W. M. Itano, D. J. Wineland, and I. C. Monroe. Experimental entanglement of four particles. Nature (London), 404:256-259, 2000.

[9] J. Clarke, A. N. Cleland, M. H. Devoret, D. Esteve, and J. M. Martinis. Science, 239:992, 1988.

[10] G. S. Agarwal. Control of decoherence and relaxation by frequency modulation of a heat bath. Phys. Rev. A, 61(1):013809, Dec 1999.

[11] G. S. Agarwal, M. O. Scully, and H. Walther. Inhibition of decoherence due to decay in a continuum. Phys. Rev. Lett., 86:4271-4274, 2001.

[12] R. Alicki et al. Optimal strategy for a single-qubit gate and the trade-off between opposite types of decoherence. Physical Review A, 70(1):10501, 2004.

[13] L. Viola and S. Lloyd. Dynamical suppression of decoherence in two-state quantum systems. Phys. Rev. A, 58:2733-2744, 1998.

[14] K. Shiokawa and D. A. Lidar. Dynamical decoupling using slow pulses: Efficient suppression of $1 / f$ noise. Phys. Rev. A, 69:030302, 2004.

[15] D. Vitali and P. Tombesi. Heating and decoherence suppression using decoupling techniques. Phys. Rev. A, 65:012305, 2001.

[16] P. Facchi and S. Pascazio. Quantum zeno and inverse quantum zeno effects. Prog. in Opt., 42:147, 2001.

[17] P. Facchi, D. A. Lidar, and S. Pascazio. Unification of dynamical decoupling and the quantum zeno effect. Phys. Rev. A, 69:0302314, 2004.

[18] P. Zanardi and S. Lloyd. Topological protection and quantum noiseless subsystems. Phys. Rev. Lett., 90:067902, 2003.

[19] P. Zanardi and M. Rasetti. Noiseless quantum codes. Phys. Rev. Lett., 79:3306, 1997.

[20] D. A. Lidar, I. L. Chuang, and K. B. Whaley. Decoherence-free subspaces for quantum computation. Phys. Rev. Lett., 81:2594, 1998.

[21] L.-A. Wu and D. A. Lidar. Creating decoherence-free subspaces using strong and fast pulses. Phys. Rev. Lett., 88(20):207902, 2002.

[22] A. G. Kofman and G. Kurizki. Quantum zeno effect on atomic excitation decay in resonators. Phys. Rev. A, 54:R3750-R3753, 1996.

[23] A. G. Kofman and G. Kurizki. Acceleration of quantum decay processes by frequent observa- 
tions. Nature (London), 405:546-550, 2000.

[24] A. G. Kofman and G. Kurizki. Universal dynamical control of quantum mechanical decay: Modulation of the coupling to the continuum. Phys. Rev. Lett., 87:270405, 2001.

[25] A. G. Kofman and G. Kurizki. Unified theory of dynamically suppressed qubit decoherence in thermal baths. Phys. Rev. Lett., 93:130406, 2004.

[26] A. G. Kofman and G. Kurizki. Theory of dynamical control of qubit decay and decoherence. IEEE Trans. Nanotechnology, 4(1):116, 2005.

[27] A. G. Kofman, G. Kurizki, and T. Opatrný. Zeno and anti-zeno effects for photon polarization dephasing. Phys. Rev. A, 63:042108, 2001.

[28] S. Pellegrin and G. Kurizki. Nonadiabatic relaxation control of qubits strongly coupled to continuum edge. Phys. Rev. A, 71:032328, 2004.

[29] M. C. Fischer, B. Gutierrez-Medina, and M. G. Raizen. Observation of the quantum zeno and anti-zeno effects in an unstable system. Phys. Rev. Lett., 87:040402, 2001.

[30] Goren Gordon, Gershon Kurizki, and Abraham G. Kofman. Universal dynamical control of local decoherence for multipartite and multilevel systems. Opt. Comm., 264:398, 2006.

[31] O. V. Prezhdo. Quantum anti-zeno acceleration of a chemical reaction. Phys. Rev. Lett., 85:4413-4417, 2000.

[32] G. S. Agarwal, M. O. Scully, and H. Walther. Accelerating decay by multiple 2 pi pulses. Phys. Rev. A, 63:044101, 2001.

[33] L. Viola, E. Knill, and S. Lloyd. Dynamical decoupling of open quantum systems. Phys. Rev. Lett., 82:2417-2421, 1999.

[34] L. Viola, E. Knill, and S. Lloyd. Dynamical generation of noiseless quantum subsystems. Phys. Rev. Lett., 85(16):3520, 2000.

[35] K. Banaszek, A. Dragan, W. Wasilewski, and C. Radzewicz. Experimental demonstration of entanglement-enhanced classical communication over a quantum channel with correlated noise. Phys. Rev. Lett., 92:257901, 2004.

[36] Antonio Di Lisi and Klaus Mølmer. Entanglement of two atomic samples by quantumnondemolition measurements. Phys. Rev. A, 66(5):052303, Nov 2002.

[37] Goren Gordon and Gershon Kurizki. Preventing multipartite disentanglement by local modulations. Phys. Rev. Lett., 97(11):110503, Sep 2006.

[38] S. Nakajima. On quantum theory of transport phenomena. Prog. Theor. Phys, 20:948, 1958. 
[39] R. Zwanzig. Ensemble Method in the Theory of Irreversibility. Journal of Chemical Physics, 33(5):1338-1341, 1964.

[40] I. Prigogine. Non-equilibrium statistical mechanics. Interscience Publ., 1962.

[41] H.P. Breuer and F. Petruccione. The theory of open quantum systems. Oxford University Press New York, 2002.

[42] Y.J. Yan and R.X. Xu. QUANTUM MECHANICS OF DISSIPATIVE SYSTEMS. Annual Review of Physical Chemistry, 56(1):187-219, 2005.

[43] N. Hashitsumae, F. Shibata, and M. Shing u. Quantal master equation valid for any time scale. Journal of Statistical Physics, 17(4):155-169, 1977.

[44] F. Shibata, Y. Takahashi, and N. Hashitsume. A generalized stochastic liouville equation. NonMarkovian versus memoryless master equations. Journal of Statistical Physics, 17(4):171-187, 1977.

[45] S. Chaturvedi and F. Shibata. Time-convolutionless projection operator formalism for elimination of fast variables. Applications to Brownian motion. Zeitschrift für Physik B Condensed Matter, 35(3):297-308, 1979.

[46] F. Shibata and T. Arimitsu. Expansion Formulas in Nonequilibrium Statistical Mechanics. Journal of the Physical Society of Japan, 49(3):891-897, 1980.

[47] G.G. Emch and G.L. Sewell. Nonequilibrium Statistical Mechanics of Open Systems. Journal of Mathematical Physics, 9(6):946-958, 1968.

[48] G. S. AGARWAL. Master equations in phase-space formulation of quantum optics. Phys. Rev., 178(5):2025-2035, Feb 1969.

[49] A. G. Kofman and G. Kurizki. Frequent observations accelerate decay: The anti-zeno effect. Z. Naturforsch. A, 56:83-90, 2001.

[50] B. Sherman, G. Kurizki, and A. Kadyshevitch. Nonclassical field-dynamics in photonic band structures - atomic-beam resonant interaction with a spatially periodic field mode. Phys. Rev. Lett., 69:1927-1930, 1992.

[51] Y. Japha and G. Kurizki. Spontaneous emission from tunneling two-level atoms. Phys. Rev. Lett., 77:2909-2912, 1996.

[52] Q. Niu and M. G. Raizen. How landau-zener tunneling takes time. Phys. Rev. Lett., 80:34913494, 1998.

[53] W. K. Wootters. Phys. Rev. Lett., 80:2245, 1998. 